\title{
ON The Bosons' Range of The Weak Interaction
}

\author{
Antonio PUCCINI \\ Neurophysiologist of Health Ministry, Naples -Italy \\ antonio_puccini@libero.it
}

\section{Abstract}

As known the Weak Nuclear Force or Weak Interaction(WI) acts between quarks (Qs) and leptons. The action of the WI is mediated by highly massive gauge bosons. How does a nuclear $\mathrm{Q}$ emit such a massive particle, approximately 16000 or 40000 times its mass? Who provides so much energy to a up Q or a down Q?

However, it must be considered that according to Quantum Mechanics it is possible to loan temporarily some energy, but to a precise and binding condition, established by the Uncertainty Principle: the higher the energy borrowed, the shorter the duration of the loan.

Our calculations show that the maximum distance these bosons can travel, i.e. the upper limit of their range, corresponds to $1.543 \cdot 10^{-15}[\mathrm{~cm}]$ for particles $\mathrm{W}^{+}$and $\mathrm{W}^{-}$and $1.36 \cdot 10^{-15}[\mathrm{~cm}]$ for $Z^{\circ}$ particles.

Keywords: Weak Interaction (WI); Quark (Q); Energy (E): Distance (d).

Date of Submission: 30 August, 2018

DOI: 10.24297/jap.v14i3.7631

ISSN: 2347-3487

Volume: 14 Issue: 3

Journal: Journal of Advances in Physics

Website: https://cirworld.com

This work is licensed under a Creative Commons Attribution 4.0 International License. 


\section{Introduction}

The Weak Nuclear Force or Weak Interaction(WI) is a short-range force, which acts within the space of an atomic nucleus. The WI acts between both Quarks (Qs) and leptons. Enrico Fermi, the one who first and alone built the entire mathematical formalism of the Neutron Decay, connected to the superb intuition of the existence of WI [1,2]. As described by Heisemberg [3] and Majorana [4], nuclear particles interact with each other by continuously exchanging another type of particle, subsequently defined as gauge boson. This is the concept of exchange forces.

Particles sensitive to WI exchange the bosons $\mathrm{W}^{+}, \mathrm{W}^{-}$or $Z^{\circ}$, discovered by Rubbia and Van der Meer in 1983. Bosons $\mathrm{W}^{+}$and $\mathrm{W}^{-}$have a mass about $80.385 \pm 0.015 \mathrm{GeV} / \mathrm{c}^{2}$, whereas the particle $\mathrm{Z}^{\circ}$ weighs about $91.1876 \pm 0.0021 \mathrm{GeV} / \mathrm{c}^{2}$.

As regards the radius of action of the WI we don't find unique data in literature: they mostly hover around $10^{-}$ ${ }^{16}[\mathrm{~cm}]$. Let's try to deepen this topic.

\section{Discussion}

According to Quantum Field Theory a particle generates a field, and the field acts on another particle. "Consider two particles rather close to each other: each of them surrounds itself with its field, which will have to act on the other. Get surrounded by own field means emit quanta of this field[5]". The quanta of action of the field are said bosons, which differ as the generated field vary, namely as the Fundamental Force taken into account vary. Thus a $\mathrm{Q}$ can emit a boson and interact with another $\mathrm{Q}$. Qs which frequently interact with each other are up $\mathrm{Q}$ and down $\mathrm{Q}$, which respectively weigh about $2 \mathrm{MeV} / \mathrm{c}^{2}$ and $5 \mathrm{MeV} / \mathrm{c}^{2}$.

To this point one wonders: how does a $\mathrm{Q}$ emit a particle so much heavier and so more energetic? For instance a boson $\mathrm{W}$, emitted by the $\mathrm{Q}$, is about 40000 times heavier than the up $\mathrm{Q}$ and 16000 times heavier than the down Q. Saying heavier is as to say more energetic, along with the Mass-Energy Equivalence Principle [6]:

$$
E=m c^{2}
$$

Then: who is giving so much energy $(E)$ to the $\mathrm{Q}$, so that it can emit a particle of so much $E$ as the W boson? This $E$ is borrowed from the Q. Quantum Mechanics (QM) allows it to do so, but at a precise and binding condition: the higher $E\left(\Delta_{E}\right)$ loaned, the sooner the loan must be paid, that is the shorter the time $\left(\Delta_{t}\right)$ of the loan. This is required by one of the pillars of the $\mathrm{QM}$, that is the Uncertainty Principle of Heisemberg concerning the energy and the time $[7,8]$ :

$$
\Delta_{E} \cdot \Delta_{t} \geq \mathrm{h}
$$

where $h$ is Planck's constant equal to $6.626 \cdot 10^{-27}$ [erg.s]. Thus the duration $(t)$ of the energetic loan is given by Eq.(2), from which we get:

$$
t=h / E
$$

The value of $E$ in Eq.(3) can be substituted by the value $m c^{2}$ of Eq.(1):

$$
t=h / m c^{2}
$$

Eq.(3) shows very clearly that the time $(t)$ and $E$ are inversely proportional. That's why the higher the $E$ loaned, the lower the period of the loan. Eq.(3), thus, as Fermi reminds us "represents the time in which the boson issued can stay in the free space. If then it is assumed that its speed is the maximum speed at which a particle can move, that is the speed of light $(c)$, it is seen that the maximum distance $(d)$ to which it can arrive, before being recalled to weld the debt, is given, as order of magnitude, by the product of time $(t)$ for the maximum speed at which the particle can move[5]":

$$
d=t c
$$


Introducing in Eq.(5) the value of $t$ expressed by (4), we get:

$$
d=\left(h / \mathrm{mc}^{2}\right) \cdot c
$$

that is:

$$
d=h / m c
$$

As Fermi says "Eq.(7), thus, unless some numerical coefficients, expresses the range of the nuclear forces[5]". Therefore let's calculate the range of the WI mediated by bosons W. At this point it is more convenient to consider the mass of the $\mathrm{W}$ boson in grams[g], then using the cgs metric system. As Feynman reminds us " 1 $\mathrm{GeV} / \mathrm{c}^{2}=1.782 \cdot 10^{-24}[\mathrm{~g}][9]^{\prime \prime}$.

Hence the mass of the boson W corresponds to:

$$
m_{\mathrm{w}}=80.385 \cdot\left(1.782 \cdot 10^{-24}[\mathrm{~g}]\right)
$$

that is:

$$
m_{\mathrm{w}}=1.4324607 \cdot 10^{-22}[\mathrm{~g}]
$$

that is about 100 times the mass of the proton. Let's substitute now this value to the $m$ of Eq.(7):

$$
d=6.626 \cdot 10^{-27}[\mathrm{erg} \cdot \mathrm{s}] /\left(1.4324607 \cdot 10^{-22}[\mathrm{~g}]\right)\left(2.9979 \cdot 10^{10}[\mathrm{~cm} / \mathrm{s}]\right)
$$

Since $1 \mathrm{erg}=\mathrm{g} \cdot \mathrm{cm} / \mathrm{s}^{2} \cdot \mathrm{cm}$, we can write:

$$
\begin{gathered}
d=6.626 \cdot 10^{-27}\left[\mathrm{~g} \cdot \mathrm{cm}^{2} / \mathrm{s}\right] / 4.2943739 \cdot 10^{-12}[\mathrm{~g} \cdot \mathrm{cm} / \mathrm{s}] \\
d=1.542949 \cdot 10^{-15}[\mathrm{~cm}]
\end{gathered}
$$

which we round to:

$$
d=1.543 \cdot 10^{-15}[\mathrm{~cm}]
$$

This is the upper limit of the range of the $\mathrm{W}$ boson.

Let's consider the other boson that mediates the WI, namely the $Z^{\circ}$ particle, whose mass would correspond to $\approx 91.1876 \mathrm{GeV} / \mathrm{c}^{2}$. Let's write this mass in $[\mathrm{g}]$ :

$$
m_{z}=91.1876 \cdot\left(1.782 \cdot 10^{-24}[\mathrm{~g}]\right)
$$

i.e.:

$$
m_{z}=1.624963 \cdot 10^{-22}[\mathrm{~g}]
$$

Also in this case we can substitute this value with the $m$ from Eq.(7):

$$
\begin{gathered}
d=6.626 \cdot 10^{-27}[\mathrm{erg} \cdot \mathrm{s}] /\left(1.624963 \cdot 10^{-22}[\mathrm{~g}]\right)\left(2.9979 \cdot 10^{10}[\mathrm{~cm} / \mathrm{s}]\right) \\
d=6.626 \cdot 10^{-27}\left[\mathrm{~g} \cdot \mathrm{cm}^{2} / \mathrm{s}\right] / 4.8714765 \cdot 10^{-12}[\mathrm{~g} \cdot \mathrm{cm} / \mathrm{s}]
\end{gathered}
$$

that is: 


$$
d=1.3601625 \cdot 10^{-15}[\mathrm{~cm}]
$$

as to say:

$$
d=1.36 \cdot 10^{-15}[\mathrm{~cm}]
$$

The latter equation delineates the upper limit of the range of the boson $Z^{\circ}$.

\section{Conclusions}

With our work we tried to give a contribution to the literature on the measurement of the maximum distance gauge bosons of the WI can travel before the time of paying back the energetic debt.

It is this short time to narrow their journey, it cannot go beyond $10^{-15}[\mathrm{~cm}]$. Namely, the upper limit of their range corresponds to $1.543 \cdot 10^{-15}[\mathrm{~cm}]$ for particles $\mathrm{W}^{+}$and $\mathrm{W}^{-}$and $1.36 \cdot 10^{-15}[\mathrm{~cm}]$ for $Z^{\circ}$ particles.

The small difference between the radius of the particle $Z^{\circ}$ and that of bosons $\mathrm{W}$ (slightly larger the latter) goes perfectly along with the known fact that the action range of a Fundamental Force is inversely proportional to the mass of the bosons the force conveys [10].

\section{References}

1) Fermi E., "Tentativo di una teoria dell'emissione dei raggi $\beta$ ",La Ricerca Scientifica,4,491-495,1933.

2) Fermi E., "Tentativo di una teoria dei raggi $\beta$ ", Il Nuovo Cimento,11,1-19, 1934.

3) Heisemberg W., "Über den Bau der Atomkerne. I", Zeitschrift fur Physik,Vol.77,1-11,1932.

4) Majorana E., "Uber die Kerntheorie", Zeitschrift fur Physik,Vol.82,137-145,1933.

5) Fermi E., "Atomi Nuclei Particelle", a cura di V. Barone, Boringhieri Ed.(Torino),140-141,2009.

6) Einstein A., "Ist die Tragheit eines Korpers von seinem Energieinhalt abhangig?", Annalen der Physik, 18, 639-641, 1905.

7) Heisemberg W., "Uber den anscauliken Inhalt der Quantentheoritschen Kinematik und Mechanik", Zeitschrift fur Physik,Vol.43,184-185,1927.

8) Puccini A., "Uncertainty Principle and Electromagnetic Waves", Journal of Electromagnetic Waves and Applications, Vol.19, N7, 885-890, 2005.

9) Feynman R.P., "QED. The Strange Theory of Light and Matter", @1985 by Richard P. Feynman; Adelphi Ed.(Torino),164,1989.

10) Yukawa H., "On the Interaction of Elementary Particles", Proceeding Phys. Math. Soc. Japan, 17, 48-57,1935. 